\title{
ARTICLE
}

\section{Shot noise generated by graphene $p-n$ junctions in the quantum Hall effect regime}

N. Kumada ${ }^{1,2}$, F.D. Parmentier ${ }^{2}$, H. Hibino ${ }^{1}$, D.C. Glattli² \& P. Roulleau ${ }^{2}$

Graphene offers a unique system to investigate transport of Dirac Fermions at $p-n$ junctions. In a magnetic field, combination of quantum Hall physics and the characteristic transport across $p-n$ junctions leads to a fractionally quantized conductance associated with the mixing of electron-like and hole-like modes and their subsequent partitioning. The mixing and partitioning suggest that a $p-n$ junction could be used as an electronic beam splitter. Here we report the shot noise study of the mode-mixing process and demonstrate the crucial role of the $p-n$ junction length. For short $p-n$ junctions, the amplitude of the noise is consistent with an electronic beam-splitter behaviour, whereas, for longer $p$ - $n$ junctions, it is reduced by the energy relaxation. Remarkably, the relaxation length is much larger than typical size of mesoscopic devices, encouraging using graphene for electron quantum optics and quantum information processing.

\footnotetext{
${ }^{1}$ NTT Basic Research Laboratories, NTT Corporation, 3-1 Morinosato-Wakamiya, Atsugi 243-0198, Japan. 2 Nanoelectronics Group, Service de Physique de I'Etat Condensé, IRAMIS/DSM (CNRS URA 2464), CEA Saclay, F-91191 Gif-sur-Yvette, France. Correspondence and requests for materials should be addressed to N.K. (email: kumada.norio@lab.ntt.co.jp).
} 
$\mathrm{n}$ graphene, owing to the linear and gapless band structure, $n$-type (electron-like) and $p$-type (hole-like) regions can adjoin without a gap in between. Investigations of charge carrier transport across such a $p-n$ junction (PNJ) have revealed unique phenomena reflecting the Dirac Fermion character in graphene, such as Klein tunneling ${ }^{1-3}$, Veselago lensing ${ }^{4}$ and snake state ${ }^{5,6}$. In the quantum Hall $(\mathrm{QH})$ effect regime under high magnetic field $B$, the conductance across a PNJ shows plateaus at $G_{\mathrm{PNJ}}=G_{0}\left|v_{1}\right|\left|v_{2}\right| /\left(\left|v_{1}\right|+\left|v_{2}\right|\right)$, where $G_{0}=e^{2} / h$ is the conductance quantum ( $h$ is Planck's constant), $v_{1}=2,6,10, \ldots$ and $v_{2}=-2$, $-6,-10, \ldots$ are the Landau level filling factor in the $n$ and $p$ regions, respectively ${ }^{7-9}$. This conductance quantization in bipolar QH states has been explained by the mixing of counter-circulating electron and hole edge modes ${ }^{10,11}$ : the current injected to the PNJ is distributed to electron and hole modes in the PNJ by the mode mixing with the ratio depending on the number of each modes, thus on $v_{1}$ and $v_{2}$, and then partitioned at the exit of the PNJ (Fig. 1b). This process gives rise to the conductance quantization at the values depending on $v_{1}$ and $v_{2}$. However, experimental study of the mode-mixing mechanism is lacking. If the mode mixing is caused by quasielastic scattering as suggested in ref. 10,11, a graphene PNJ acts as a beam splitter of electrons and holes. A better understanding of these properties is a crucial step towards the development of electron quantum optics experiments in graphene; beam splitters together with edge states are key components for electronic interferometry ${ }^{12-14}$.

Shot noise measurements can provide insight into the mode-mixing mechanism (Supplementary Figs 1 and 4): when the electron and hole modes biased by $V_{\text {sd }}$ are mixed, the energy distribution in the $\mathrm{PNJ} f_{\mathrm{PNJ}}(E)$ becomes out-of-equilibrium and the subsequent partitioning of the modes gives rise to the shot noise. If the mode mixing is quasielastic, $f_{\mathrm{PNJ}}(E)$ is a double-step function. At zero temperature, the shot noise generated by the partitioning of the modes with double-step $f_{\mathrm{PNJ}}(E)$ is expected to be (ref: 10, Supplementary Note 1),

$$
S_{\mathrm{I}}=2 e G_{0} \frac{\left(\left|v_{1}\right|\left|v_{2}\right|\right)^{2}}{\left(\left|v_{1}\right|+\left|v_{2}\right|\right)^{3}} V_{\mathrm{sd}}=2 e \frac{\left|v_{1}\right|\left|v_{2}\right|}{\left(\left|v_{1}\right|+\left|v_{2}\right|\right)^{2}} G_{\mathrm{PNJ}} V_{\mathrm{sd}}
$$

characterized by the Fano factor $F=S_{\mathrm{I}} / 2 e I$, yielding $F=0.25$ for $\left(v_{1}, v_{2}\right)=(2,-2)$. Energy losses towards external degrees of freedom can drive $f_{\mathrm{PNJ}}(E)$ towards a Fermi distribution with a chemical potential $e V_{\text {sd }} / 2$ (Fig. 1c) ${ }^{15-17}$, causing the noise (and thus the Fano factor) to vanish as the carrier dwell time in the PNJ becomes larger than the energy relaxation time (ref: 18). Inelastic processes between modes in the PNJ may occur, causing $f_{\mathrm{PNJ}}(E)$ to relax towards a Fermi distribution with a finite temperature $T_{\text {eff }}\left(V_{\text {sd }}\right)$ given by the balance between the Joule power dissipated in the PNJ and the heat flowing along the outgoing electronic channels ${ }^{10,19}$ (Supplementary Note 1). In this case, the Fano factor becomes $\tilde{F}=(3 F)^{\frac{1}{2}} / \pi\left[\tilde{F} \sim 0.28\right.$ for $\left.\left(v_{1}, v_{2}\right)=(2,-2)\right]$. Note that standard transport measurements yield the same value of $G_{\mathrm{PNJ}}$ for all cases, and thus cannot distinguish them.

In the following, we investigate the evolution of $f_{\mathrm{PNJ}}(E)$ with carrier dwell time in the PNJ, which can be tuned by changing the length of the PNJ. We demonstrate that the amplitude of the noise is consistent with an electronic beam-splitter behaviour when PNJ is short and the energy losses are negligible.

\section{Results}

Measurement set-up. We obtained bipolar graphene devices using a top gate covering half of the graphene (Fig. 1a); the carrier type in the gated region can be tuned by the gate voltage $V_{\mathrm{G}}$, while that in the ungated region is fixed to electron by the doping (Methods). Therefore, the PNJ is formed at the interface between

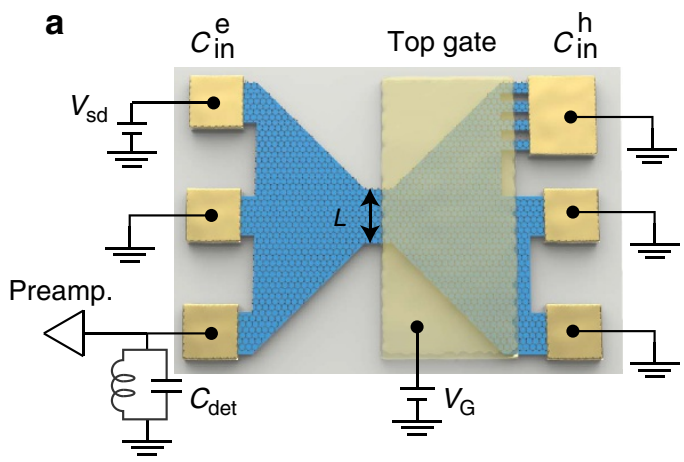

b
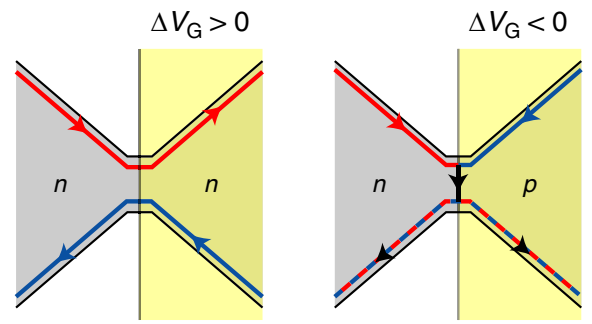

C

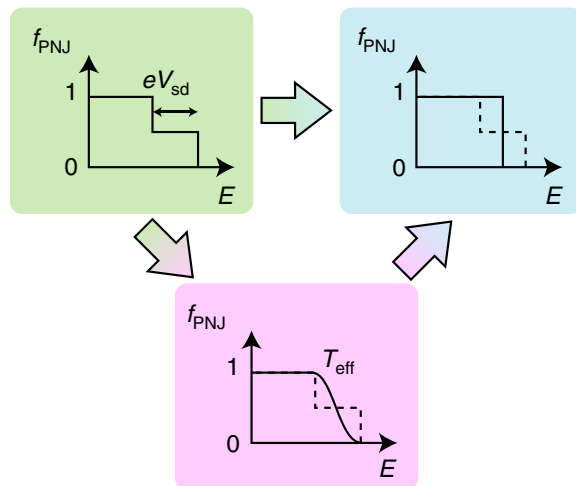

Figure 1 | Schematic of the device. (a) The top gate covers the right half of the graphene. In the ungated region, the carrier type is electron and the density is fixed at about $5 \times 10^{11} \mathrm{~cm}^{-2}$. In the gated region, the carrier type can be changed to hole by applying negative gate voltage $V_{G}$. A sourcedrain bias $V_{\text {sd }}$ is applied to either $C_{\text {in }}^{e}$ or $C_{\text {in }}^{h}$ and the noise is measured on $C_{\text {det. }}$. The upperright contact is comb shaped to achieve a good contact to the $p$ region (Supplementary Methods). (b), QH edge modes for the unipolar (left) and bipolar regimes (right). (c), $f_{\mathrm{PN} J}(E)$ in the quasielastic case (left). In the presence of energy relaxation towards external degrees of freedom, $f_{\mathrm{PNJ}}(E)$ becomes a Fermi distribution at base temperature (right). Additional inelastic scattering between modes in the PNJ may drive towards a Fermi distribution at finite temperature $T_{\text {eff }}$ (bottom), which can then relax towards a cold Fermi distribution.

the gated and ungated regions when the carrier type in the gated region is hole for $\Delta V_{\mathrm{G}} \equiv V_{\mathrm{G}}-V_{\mathrm{CNP}}<0 \quad\left(V_{\mathrm{CNP}}\right.$ is the gate voltage at the charge neutrality point). We prepared five samples with different interface lengths $L=5,10,20,50$, and $100 \mu \mathrm{m}$. The direction of $B$ is chosen so that electron and hole modes from the ohmic contacts $C_{\text {in }}^{\mathrm{e}}$ and $C_{\mathrm{in}}^{\mathrm{h}}$, respectively, merge at the PNJ. For the noise measurement, $V_{\mathrm{sd}}$ is applied to either $C_{\text {in }}^{\mathrm{e}}$ or $C_{\mathrm{in}}^{\mathrm{h}}$ and the noise is detected on $C_{\text {det }}$ (Fig. 1a; Supplementary Methods and Supplementary Fig. 5). Magnetic fields up to $B=16 \mathrm{~T}$ have been applied. The base temperature is $T=4.2 \mathrm{~K}$.

Detection of shot noise generated at PNJ. The inset of Fig. 2 shows the reflection of the averaged current from $C_{\text {in }}^{\mathrm{e}}$ to $C_{\mathrm{det}}$ in 


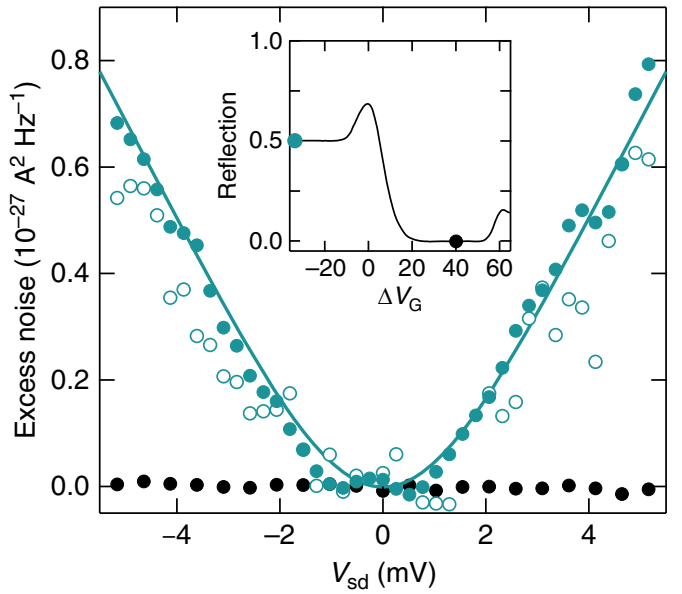

Figure $\mathbf{2}$ | Shot noise generated by $\boldsymbol{p}-\boldsymbol{n}$ junction. Excess noise $\Delta S_{\text {, }}$ of the sample with $L=50 \mu \mathrm{m}$ as a function of $V_{\mathrm{sd}}$ for the bipolar $\mathrm{QH}$ state at $\left(v_{\text {ug }}, v_{\mathrm{g}}\right)=(2,-2)$ (cyan circles) and the unipolar $\mathrm{QH}$ state at $\left(v_{\mathrm{ug}}, v_{\mathrm{g}}\right)=$ $(2,2)$ (black circles). For the solid and open circles, $V_{s d}$ is applied to $C_{\text {in }}^{e}$ and $C_{\text {in, }}^{h}$, respectively. The solid trace is the result of a fit using equation (2). Inset: reflection of the average current from $C_{\text {in }}^{e}$ to $C_{\text {det }}$ as a function of $\Delta V_{G}$. The cyan and black circles represent the $\Delta V_{\mathrm{G}}$ at which the noise is measured.

the sample with $L=50 \mu \mathrm{m}$. The magnetic field is $B=10 \mathrm{~T}$, at which the filling factor in the ungated region is fixed at $v_{\mathrm{ug}}=2$. When the bipolar $\mathrm{QH}$ state at $\left(v_{\mathrm{ug}}, v_{\mathrm{g}}\right)=(2,-2)$ is formed for $\Delta V_{\mathrm{G}}<-10 \mathrm{~V}$, the current injected from $C_{\mathrm{in}}^{\mathrm{e}}$ is partitioned equally to the electron and hole modes at the exit of the PNJ, yielding a reflection of $1 / 2$. A current noise $S_{\mathrm{I}}$ appears in this regime. As $V_{\text {sd }}$ applied to $C_{\text {in }}^{e}$ is increased, the excess noise $\Delta S_{\mathrm{I}} \equiv S_{\mathrm{I}}-S_{\mathrm{I}}\left(V_{\text {sd }}=0\right)$ increases (solid cyan circles in Fig. 2). $\Delta S_{\mathrm{I}}$ approaches linear behaviour for $e V_{\mathrm{sd}}>k_{\mathrm{B}} T$, characteristic of the shot noise. A similar signal appears when $V_{\text {sd }}$ is applied to $C_{\text {in }}^{h}$ (open cyan circles). In the unipolar QH state at $\left(v_{\mathrm{ug}}, v_{\mathrm{g}}\right)=(2,2)$ for $20<\Delta V_{\mathrm{G}}<50 \mathrm{~V}$, on the other hand, the shot noise is zero (solid black circles), proving that the shot noise is indeed generated at the PNJ.

Quantitatively, we extracted $F$ by fitting $\Delta S_{\mathrm{I}}$ as a function of $V_{\text {sd }}$ using the relation including temperature broadening ${ }^{20}$ :

$$
\Delta S_{\mathrm{I}}=2 e F G_{\mathrm{PNJ}} V_{\mathrm{sd}}\left[\operatorname{coth}\left(\frac{e V_{\mathrm{sd}}}{2 k_{\mathrm{B}} T}\right)-\frac{2 k_{\mathrm{B}} T}{e V_{\mathrm{sd}}}\right]
$$

where $G_{\mathrm{PNI}}$ is obtained by average current measurements. The fit yields $F=0.015$, which is one order of magnitude smaller than $F=0.25$ expected for the noise from the double-step energy distribution. This indicates that $f_{\mathrm{PNJ}}(E)$ evolves during the charge propagation for $L=50 \mu \mathrm{m}$, reducing the shot noise.

Evolution of noise amplitude with PNJ length. The evolution of $f_{\mathrm{PNJ}}(E)$ can be investigated using samples with different $L$. Figure $3 \mathrm{a}$ shows the results of the noise measurement in the bipolar QH state at $\left(v_{\mathrm{ug}}, v_{\mathrm{g}}\right)=(2,-2)$ for the five samples with $L$ between 5 and $100 \mu \mathrm{m}$. The data show that the shot noise decreases with increasing $L$ and almost disappears at $L=100 \mu \mathrm{m}$ (Fig. 3b), indicating that $f_{\mathrm{PNJ}}(E)$ relaxes to the thermal equilibrium through interactions with external degrees of freedom. An exponential fit of the data yields a relaxation length $L_{0}=15 \mu \mathrm{m}$. The extrapolation to $L=0$ gives $F \sim 0.27$, consistent with the limit of quasielastic scattering $F=0.25$. Furthermore, the decrease is well reproduced by a model gradually coupling the modes propagating in the PNJ to cold external states (Supplementary Note 1 and Supplementary Fig. 2). Note that we a
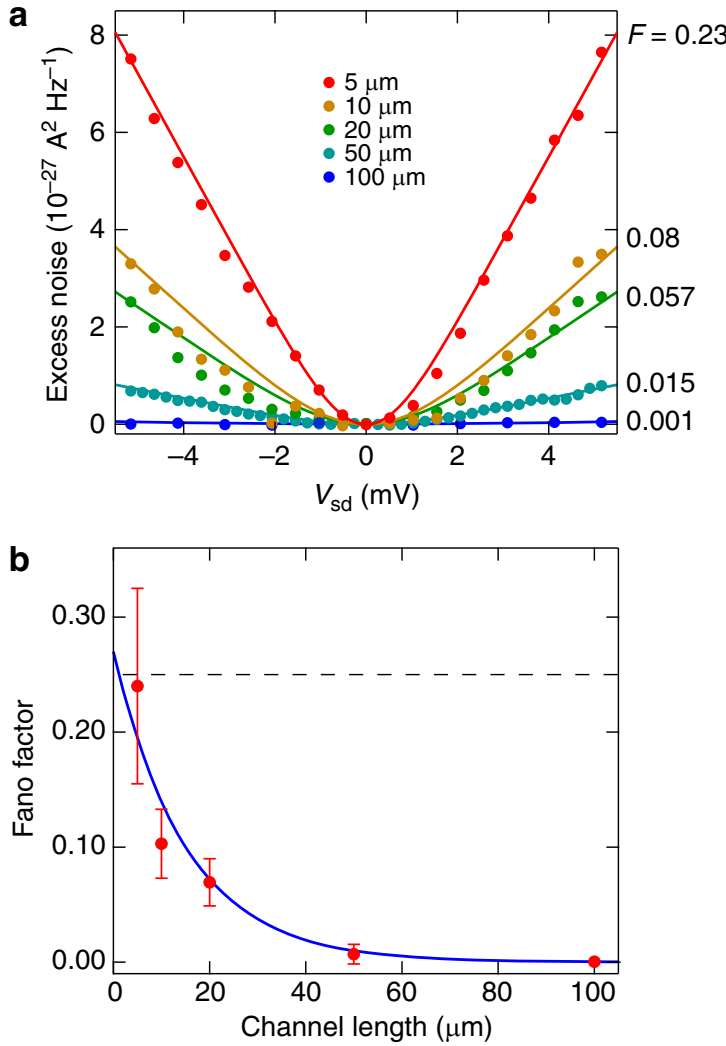

Figure 3 | Shot noise as a function of $\boldsymbol{p}$-n junction length. (a) $\Delta S_{\mid}$in the bipolar QH state at $\left(v_{\mathrm{ug}}, v_{\mathrm{g}}\right)=(2,-2)$ as a function of $V_{\mathrm{sd}}$ for the five samples with $L$ between 5 and $100 \mu \mathrm{m}$. All data are taken at $B=10 \mathrm{~T}$. Lines are results of the fit using equation (2), by which the values of $F$ (indicated on the right-hand side of the figure) are obtained. (b), $F$ as a function of $L$. The error bars represent the s.d. of the extracted $F$ for different values of $B$ and $\Delta V_{G}$ in the bipolar $\mathrm{QH}$ state (an example is shown in Fig. $4 \mathrm{c}$ ). An exponential fit (blue curve) yields a relaxation length $L_{0}=15 \mu \mathrm{m}$. The dashed horizontal line represents the expected value $F=0.25$ for the double-step $f_{\mathrm{PNJ}}(E)$.

are not able to observe whether inelastic scattering occurs inside the PNJ, because of the large error bars explained below. An important implication of the results is that, within the typical scale of usual mesoscopic devices $(<1 \mu \mathrm{m})$, the energy loss towards external degrees of freedom is negligible and the current channels in the PNJ can be regarded as an isolated system.

Fluctuation of noise. We further investigate the properties of the PNJ focusing on the energy relaxation mechanism by measuring the shot noise for a wide range of $B$ and $\Delta V_{\mathrm{G}}$. We identify the electronic states in the gated and ungated regions as a function of $B$ and $\Delta V_{\mathrm{G}}$ by a low-frequency current measurement from $C_{\text {in }}^{\mathrm{e}}$ to $C_{\text {det }}$ (Fig. 4a) and then investigate the relation between those states and the noise. The electronic state in the ungated region depends only on $B$ and the $v_{\mathrm{ug}}=2 \mathrm{QH}$ state is formed for $B>4 \mathrm{~T}$. In the gated region, the non- $\mathrm{QH}$ states at $v_{\mathrm{g}}=8,4,0$ and -4 appear as a current peaks. The bipolar $\mathrm{QH}$ state at $\left(v_{\mathrm{ug}}, v_{\mathrm{g}}\right)=$ $(2,-2)$ is formed for $B>4 \mathrm{~T}$ and between $v_{\mathrm{g}}=0$ and -4 (the region indicated by dashed lines), in which the current is almost constant, consistent with the quantized conductance ${ }^{7-9}$. The shot noise in the sample with $L=10 \mu \mathrm{m}$ becomes small (Fig. 4b) when either or both ungated and gated regions are in a non-QH state. This confirms that shot noise is generated by the $\mathrm{PNJ}$ in a well-developed bipolar QH state. Within the bipolar QH 
a
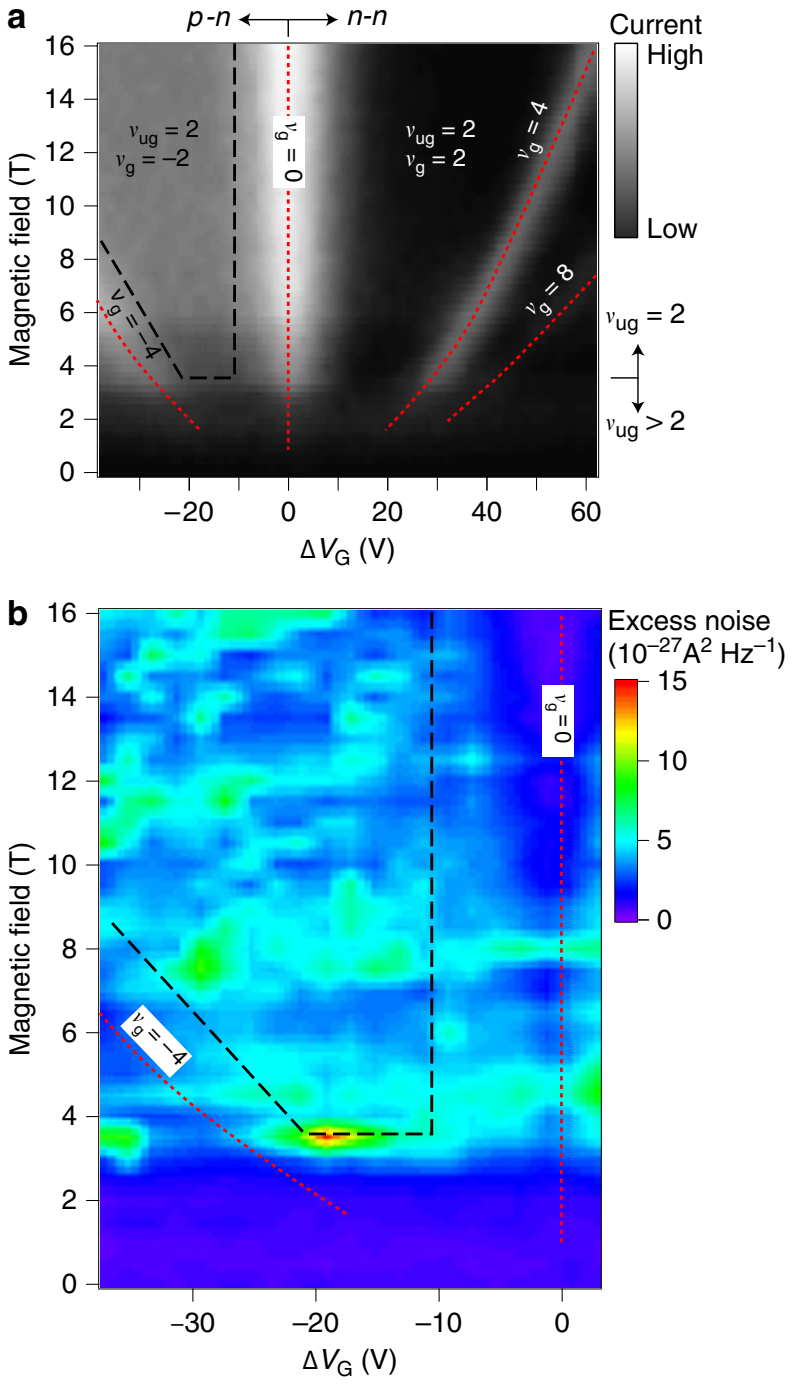

C

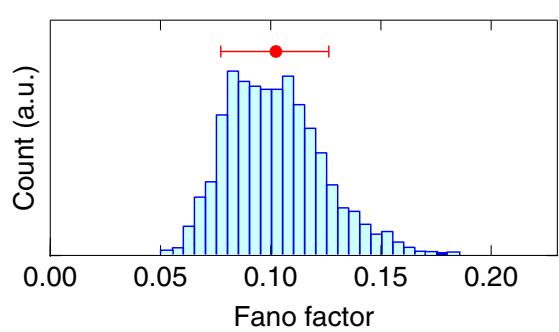

Figure 4 | Fluctuations of shot noise. (a) Grey scale plot of the current from $C_{\text {in }}^{\text {e to }} C_{\text {det }}$ at $2 \mathrm{kHz}$ for the sample with $L=10 \mu \mathrm{m}$. The current is measured through the resonator and the amplifier. Black dashed lines indicate the region for the bipolar $\mathrm{QH}$ state at $\left(v_{\mathrm{ug}}, v_{\mathrm{g}}\right)=(2,-2)$. Red dotted lines represent the non- $\mathrm{QH}$ state in the gated region at $v_{\mathrm{g}}=-4,0$, 4 and 8. (b) Colour scale plot of $\Delta S_{1}$ for the sample with $L=10 \mu \mathrm{m}$ as a function of $\Delta V_{\mathrm{G}}$ and $B$. The applied current is fixed at $400 \mathrm{nA}$, which corresponds to $V_{\mathrm{sd}}=5.2 \mathrm{mV}$ in the bipolar $\mathrm{QH}$ state at $\left(v_{\mathrm{ug}}, v_{\mathrm{g}}\right)=(2,-2)$. The black dashed and red dotted lines correspond to those in $\mathbf{a}$ (c) Histogram of the Fano factor within the bipolar QH state. The red dot and bar represent the mean value and the s.d., which correspond to the data point and the error bar in Fig. 3b, respectively.

state, the shot noise fluctuates largely, depending on $B$ and $\Delta V_{\mathrm{G}}$. This noise fluctuation cannot be ascribed to $G_{\mathrm{PNJ}}$, which is almost constant in the bipolar QH state. Furthermore, since the noise is generated in the well-developed $\mathrm{QH}$ state, the existence of multiple noise sources is unlikely. These facts indicate that the noise fluctuation is due to the fluctuation of the energy relaxation rate, which induces the fluctuation of Fano factor. Figure $4 \mathrm{c}$ shows the histogram of the Fano factor in the bipolar $\mathrm{QH}$ state calculated using equation (2). The s.d. is about $50 \%$ of the mean value.

\section{Discussion}

The random variation of the energy relaxation rate as a function of $B$ and $\Delta V_{\mathrm{G}}$ suggest that localized states in bulk graphene play a main role for the energy relaxation. Energy in the PNJ can escape to the bulk graphene through Coulomb interaction with localized states: high frequency potential fluctuations in the PNJ, which is the source of the shot noise, are dissipated in the localized states. Since the energy level and the profile of the localized states depend on $B$ and $\Delta V_{\mathrm{G}}$, fluctuations of the relaxation rate can be induced. On the other hand, the average current through the PNJ, which merely reflects the transmission coefficient, is hardly affected by the localized states. Note that a simple model of interaction with two-dimensional phonons in the PNJ fails to quantitatively reproduce our observations (Supplementary Note 1 and Supplementary Fig. 3). It is reported that the electron-phonon coupling is expected to be vanishingly small in usual unipolar edge channels ${ }^{21-23}$. To understand the energy relaxation length quantitatively, detailed analysis including interactions with phonons and any other possible mechanisms for the energy relaxation is necessary.

In conclusion, we showed that the mode mixing at PNJ in graphene bipolar $\mathrm{QH}$ states leads to non-equilibrium $f_{\mathrm{PNJ}}(E)$, generating shot noise. For a short $\mathrm{PNJ}(L<<15 \mu \mathrm{m})$, the energy loss towards external states is negligible and the noise is consistent with a quasielastic mode mixing. This suggests that a graphene PNJ can act as a beam splitter. Since $15 \mu \mathrm{m}$ is much larger than typical length scale of mesoscopic devices, our results encourage using graphene for electron quantum optics experiments and quantum information.

\section{Methods}

Device fabrication. We prepared a graphene wafer by thermal decomposition of a $6 \mathrm{H}-\mathrm{SiC}(0001)$ substrate. $\mathrm{SiC}$ substrates were annealed at around $1,800^{\circ} \mathrm{C}$ in $\mathrm{Ar}$ at a pressure of $<100$ torr. For the fabrication of devices, graphene was etched in an $\mathrm{O}_{2}$ atmosphere. After the etching, the surface was covered with 100 -nm-thick hydrogen silsesquioxane (HSQ) and 60-nm-thick $\mathrm{SiO}_{2}$ insulating layers. As a result of doping from the SiC substrate and the HSQ layer, graphene has $n$-type carriers with the density of about $5 \times 10^{11} \mathrm{~cm}^{-2}$. The width of the PNJ roughly corresponds to the thickness of the insulating layers and estimated to be $200 \mathrm{~nm}$ at most. In the $\mathrm{QH}$ effect regime, because of the Landau level quantization, the width becomes smaller with $B$. An important advantage of the $\mathrm{SiC}$ graphene is its size: it is single domain for $1 \mathrm{~cm}^{2}$, allowing us to investigate the effect of PNJ length.

Noise measurement. For the noise measurement, the current noise is converted into voltage fluctuations across one $2.5 \mathrm{k} \Omega$ resistor in series with the sample. A $500-\mathrm{kHz}$ bandwidth $3-\mathrm{MHz}$ tank circuit combined with a homemade cryogenic amplifier is used. After further amplification and digitization, the autocorrelation voltage noise spectra is calculated in real-time by a computer. Accurate calibration of the noise is done using Johnson-Nyquist noise that relies on the quantification of the resistance at $v=2$ and the temperature of the system.

\section{References}

1. Katsnelson, M. I., Novoselov, K. S. \& Geim, A. K. Chiral tunnelling and the Klein paradox in graphene. Nat. Phys. 2, 620-625 (2006).

2. Young, A. F. \& Kim, P. Quantum interference and Klein tunnelling in graphene heterojunctions. Nat. Phys. 5, 222-226 (2009).

3. Rickhaus, P. et al. Ballistic interferences in suspended graphene. Nat. Commun 4, 2342 (2013).

4. Cheianov, V. V., Falko, V. \& Alschuler, B. L. The focusing of electron flow and a Veselago lens in graphene p-n junctions. Science 315, 1252-1255 (2007).

5. Carmier, P., Lewenkopf, C. \& Ullmo, D. Graphene p-n junction in a strong magnetic field: a semiclassical study. Phys. Rev. B 81, 241406 (2010). 
6. Williams, J. R. \& Marcus, C. M. Snake states along graphene p-n junctions. Phys. Rev. Lett. 107, 046602 (2011).

7. Williams, J. R., DiCarlo, L. \& Marcus, C. M. Quantum Hall effect in a gate-controlled p-n junction of graphene. Science 317, 638-641 (2007)

8. Özyilmaz, B. et al. Electronic transport and quantum Hall effect in bipolar graphene p-n-p junctions. Phys. Rev. Lett. 99, 166804 (2007).

9. Lohmann, T., von Klitzing, K. \& Smet, J. H. Four-terminal magneto-transport in graphene $\mathrm{p}-\mathrm{n}$ junctions created by spatially selective doping. Nano Lett. $\mathbf{9}$, 1973-1979 (2009).

10. Abanin, D. A. \& Levitov, L. S. Quantized transport in graphene p-n junctions in magnetic field. Science 317, 641-643 (2007)

11. Long, W., Sun, Q. \& Wang, J. Disorder-induced enhancement of transport through graphene p-n junctions. Phys. Rev. Lett. 101, 166806 (2008)

12. Ji, Y. et al. An electronic Mach-Zehnder interferometer. Nature 422, 415-418 (2003).

13. Neder, I. et al. Interference between two indistinguishable electrons from independent sources. Nature 448, 333-337 (2007).

14. Roulleau, P. et al. Direct measurement of the coherence length of edge states in the integer quantum Hall regime. Phys. Rev. Lett. 100, 126802 (2008).

15. Altimiras, C. et al. Non-equilibrium edge-channel spectroscopy in the integer quantum Hall regime. Nat. Phys. 6, 34-39 (2010).

16. le Sueur, H. et al. Energy relaxation in the integer quantum Hall regime. Phys. Rev. Lett. 105, 056803 (2010).

17. Altimiras, C. et al. Tuning energy relaxation along quantum Hall channels. Phys. Rev. Lett. 105, 226804 (2010).

18. Texier, C. \& Büttiker, M. Effect of incoherent scattering on shot noise correlations in the quantum Hall regime. Phys. Rev. B 62, 7454-7458 (2000)

19. Jezouin, S. et al. Quantum limit of heat flow across a single electronic channel. Science 342, 601-604 (2013).

20. Blanter, Y. M. \& Büttiker, M. Shot noise in mesoscopic conductors. Phys. Rep. 336, 1-166 (2000)

21. Martin, T. \& Feng, S. Suppression of scattering in electron transport in mesoscopic quantum Hall systems. Phys. Rev. Lett. 64, 1971 (1990).

22. Granger, G., Eisenstein, J. P. \& Reno, J. L. Observation of chiral heat transport in the quantum hall regime. Phys. Rev. Lett. 102, 086803 (2009).
23. Nam, S.-G., Hwang, E. H. \& Lee, H.-J. Thermoelectric detection of chiral heat transport in graphene in the quantum Hall regime. Phys. Rev. Lett. 110, 226801 (2013).

\section{Acknowledgements}

We acknowledge funding from the ERC Advanced Grant 228273 MeQuaNo and the ANR MetroGraph grant. We are grateful to S. Tanabe, P. Jacques and M. Ueki for experimental support.

\section{Author contributions}

N.K. and P.R. performed the experiments. N.K., F.D.P., P.R. and D.C.G. analysed the data and wrote the manuscript. H.H. grew the wafer.

\section{Additional information}

Supplementary Information accompanies this paper at http://www.nature.com/ naturecommunications

Competing financial interests: The authors declare no competing financial interests.

Reprints and permission information is available online at http://npg.nature.com/ reprintsandpermissions/

How to cite this article: Kumada, N. et al. Shot noise generated by graphene $p-n$ junctions in the quantum Hall effect regime. Nat. Commun. 6:8068 doi: 10.1038/ncomms9068 (2015).

\section{(c) (1)}

This work is licensed under a Creative Commons Attribution 4.0 International License. The images or other third party material in this article are included in the article's Creative Commons license, unless indicated otherwise in the credit line; if the material is not included under the Creative Commons license, users will need to obtain permission from the license holder to reproduce the material. To view a copy of this license, visit http://creativecommons.org/licenses/by/4.0/ 